\title{
A Novel Miniaturized Electromagnetic Bandgap Structure and Its Effects on Signal Integrity and Electromagnetic Emission
}

\author{
Zhaowen Yan $^{1}$ and Yansheng Wang ${ }^{2}$ \\ ${ }^{1}$ School of Electronic and Information Engineering, Beihang University, No. 37 Xueyuan Road, Haidian District, Beijing 100191, China \\ ${ }^{2}$ EMC Laboratory, Missouri University of Science and Technology, 4000 Enterprise Drive, Rolla, MO 65401, USA \\ Correspondence should be addressed to Zhaowen Yan; yanzhaowen@buaa.edu.cn
}

Received 20 January 2013; Revised 25 April 2013; Accepted 29 April 2013

Academic Editor: Tat Yeo

Copyright (c) 2013 Z. Yan and Y. Wang. This is an open access article distributed under the Creative Commons Attribution License, which permits unrestricted use, distribution, and reproduction in any medium, provided the original work is properly cited.

\begin{abstract}
A miniaturized planar electromagnetic bandgap (EBG) structure with embedded meander line as bridge is proposed in this paper. The dimensions of the unit cell of the proposed EBG structure are $15 \mathrm{~mm} \times 15 \mathrm{~mm} \times 0.36 \mathrm{~mm}$. Simulation and measurement of the noise transmission coefficient $\left(S_{21}\right)$ have been performed for the structure, and good agreement can be seen. According to the measured results, the $-30 \mathrm{~dB}$ noise suppression bandwidth ranges from 0.97 to $21.54 \mathrm{GHz}$, which cover almost the whole noise band in ultrawideband applications. Moreover, its effects on signal integrity and electromagnetic emission are also analyzed.
\end{abstract}

\section{Introduction}

According to the reports released by International Technology Roadmap of Semiconductors (ITRS) in 2008 [1], electronic systems are driven in the direction of higher operating frequency, lower voltage levels, smaller size, lower cost, and faster transient current for ICs at high frequency. With these increasing trends causing electronic systems more vulnerable to noise that may be generated from either outside or inside the system itself, signal integrity (SI) and power integrity (PI) are among the major concerns in the power distribution network (PDN) design process all through chip, package, and board levels. Simultaneous switching noise (SSN), also referred to as ground bounce noise (GBN), resulting from a large number of drivers switching between high and low voltage levels simultaneously has particular relevance since it can propagate between the power/ground plane pairs, thus affecting both signal and power integrity of the whole system.

Generally speaking, there are two possible methods to reduce SSN [2]. One is to employ decoupling capacitors at chip, package, or PCB levels to keep the PDN impedance very low in a wide frequency range, except at dc. However, the decoupling capacitors are band limited and only effective below the self-resonance frequency. The other is to adopt isolation approaches among which electromagnetic bandgap (EBG) structures [2-8] are of great interest to keep part of the PDN at high impedance to attenuate SSN propagation. Up to now, various EBG structures for SSN suppression have been investigated. The planar EBG structures, compared with the mushroom-like ones, are more practicable for their low cost in the printed circuit board (PCB) fabrication process. At present, planar EBG structures applied to SSN suppression are being improved toward two directions [2]. One is stopband bandwidth enhancement due to SSN covering an increasing frequency range. The other one is miniaturization techniques because of the trends of systems in PCB, systems in package and systems on chip.

For PCB level application, the size of a unit cell is usually $30 \mathrm{~mm} \times 30 \mathrm{~mm}$ [4-7]. Reference [4] proposes a novel power/ground planes design using a low-period coplanar EBG structure (LPC-EBG) with the broadband frequency range from $600 \mathrm{Mz}$ to $4.6 \mathrm{GHz}$. The dimension of the substrate is $90 \mathrm{~mm} \times 90 \mathrm{~mm}$ with $0.4 \mathrm{~mm}$ thickness. In [5], a novel L-bridged electromagnetic bandgap (EBG) power/ ground planes is proposed with superwideband suppression of the ground bounce noise (GBN) from $600 \mathrm{Mz}$ to $4.6 \mathrm{GHz}$. The dimension of nine square unit cells is $90 \mathrm{~mm} \times$ $90 \mathrm{~mm} \times 0.4 \mathrm{~mm}$. In [6], a novel array design etching electromagnetic bandgap (EBG) structure is proposed and 
the suppressed noise level can be below $55 \mathrm{~dB}$ in the ultrawideband (UWB) frequency range from $244 \mathrm{MHz}$ to $20 \mathrm{GHz}$. The EBG structure with six cells on the power plane has the unit size of $30 \times 30 \mathrm{~mm}$, and the EBG structure in two cells pattern etched on the ground plane has the unit size of $60 \times$ $60 \mathrm{~mm}$. In [7], a novel planar electromagnetic bandgap (EBG) structure for suppressing simultaneous switching noise (SSN) is presented. The novel design is to use meander lines to increase the effective inductance of EBG patches. A $-28 \mathrm{~dB}$ suppression depth starting at $250 \mathrm{MHz}$ and extending to $12 \mathrm{GHz}$ is achieved experimentally, but the size of the unit cell is $30 \mathrm{~mm} \times 30 \mathrm{~mm}$.

However, planar EBG structures with miniaturized unit cell are increasingly in demand $[8,9]$. In [8], a novel planar EBG configuration made of smaller unit cell with multiple slits is introduced to provide low onset frequency and wide stopband $(0.9-3.5 \mathrm{GHz})$ with low impedance of the order of less than $1 \Omega$. Unit cell dimensions of the proposed design are just $15.2 \mathrm{~mm} \times 15.2 \mathrm{~mm}$. In [9], a new planar EBG with smaller unit cells is presented to provide low onset frequency and ultrawide stopband for noise suppression. A combination of low- and high-frequency EBG configurations, with newly designed unit cell incorporating Z-shaped slits and double slits, provides ultrawideband noise suppression $(1-40 \mathrm{GHz})$. The low-frequency unit cell dimensions are $1.6 \times 1.6 \mathrm{~cm}$, and the high-frequency EBG unit cell is $0.78 \times 0.78 \mathrm{~cm}$. The hybrid EBG is created by cascading $2 \times 2$ low-frequency unit cells with $4 \times 4$ high-frequency unit cells. This hybrid EBG structure shows better noise suppression.

According to the existing literature described, in order to achieve a higher suppression bandwidth, the EBG size is usually for the $30 \mathrm{~mm} \times 30 \mathrm{~mm}$. In smaller unit cell size, the suppression bandwidth is generally narrow, and if you want to reach a higher suppression bandwidth, a low- and high-frequency hybrid EBG configuration can achieve this function.

Based on the idea of [7], a planar electromagnetic bandgap (EBG) structure with embedded meander bridge for broadband suppression of simultaneous switching noise (SSN) in power/ground plane pairs is proposed [10]. Simulation results show that the $-30 \mathrm{~dB}$ suppression bandwidth of the novel structure is from $500 \mathrm{MHz}$ to $14.5 \mathrm{GHz}$. Effects of the proposed EBG structure on signal integrity (SI) are investigated, and the methods for improving SI are also concluded. Because the structure has the larger size $(90 \mathrm{~mm}$ by $90 \mathrm{~mm}$ with $0.4 \mathrm{~mm}$ thickness), its suppression bandwidth and suppression depth are not large enough.

By changing the number and size of embedded meander bridges and the size and thickness of the substrate, the suppression bandwidth and suppression depth can be proved. Based on the idea, this paper proposes a miniaturized EBG structure which can suppress SSN below $-30 \mathrm{~dB}$ in a wide frequency range from 0.97 to $21.54 \mathrm{GHz}$ by using $3 \times 3$ unit cells (the size of each unit cell is $15 \mathrm{~mm} \times 15 \mathrm{~mm}$ ). Because the planar EBG structures are highly attractive from the manufacturing perspective, its application in signal integrity and the effects on electromagnetic emission are also studied. This structure has two advantages of the wide suppression bandwidth and miniaturization.
This paper is organized as follows. Section 2 describes the design principles, detailed dimensions, and simulation results of SSN suppression performance for the proposed miniaturized planar EBG structure. Section 3 provides measured results of SSN suppression performance of the fabricated EBG structure to verify the design's correctness. Section 4 analyzes its application in signal integrity. Section 5 tests the electromagnetic emission of the EBG board, which is compared with the measured results of the solid reference board. Finally, Section 6 concludes this paper.

\section{Structure Design}

2.1. Design Principles. For planar EBG structures, the pattern usually designed directly on the power or ground planes is built by periodic patches that connect to each other by a slender microstripline called bridge. Two dimensional (2D) as the planar EBG structure is, a simplified 1D circuit model can be adopted to analyze, explains and predict the structure's SSN suppression performance. According to [2], 1D planar EBG structure shown in Figure 1(a) can be modeled as a simple circuit model shown in Figure 1(b). Each patch is modeled as two cascading $\pi$-models, which are described by $L_{p}$ and $C_{p}$, and half of the bridge inductance is represented by $L_{b}$. With these parameters, the lower side and upper side cutoff frequencies $\left(f_{L}\right.$ and $\left.f_{H}\right)$ for the $1 \mathrm{D}$ planar EBG structure can be derived as follows:

$$
\begin{gathered}
f_{L}=\frac{1}{2 \pi} \sqrt{\frac{1}{C_{p} L_{b}}}, \\
f_{H}=\frac{1}{2 \pi} \sqrt{\frac{1}{C_{p} L_{p}}} .
\end{gathered}
$$

Based on the two equations, patch capacitance $(4 \times$ $C_{p}$ ) should be reduced in order to enhance the stopband bandwidth (the difference between $f_{H}$ and $f_{L}$ ). Meanwhile, bridge inductance $\left(2 \times L_{b}\right)$ should be increased properly so as to decrease the lower side cutoff frequency. In accordance with these requirements, the proposed miniaturized planar EBG structure enlarges the etched area of the patch to reduce patch capacitance and introduces meander microstripline as the bridge to increase bridge inductance.

2.2. Detailed Dimensions. To describe the design clearly, the topologies and parameters of the proposed EBG structure have been shown from Figure 2 to Figure 4. Figures 2(a) and 2 (b) show the $3 \mathrm{D}$ view and top view of our EBG structure. The power plane of the structure is etched into EBG pattern with 9 (3 by 3) unit cells, while the ground plane is kept solid. The dielectric between the power/ground plane pairs is common FR- 4 with $\varepsilon_{r}=4.3$ and a loss tangent of 0.02 , and its dimensions are $45 \mathrm{~mm} \times 45 \mathrm{~mm} \times 0.36 \mathrm{~mm}$. Figures 3(a) and 3 (b) show the $3 \mathrm{D}$ view and top view of the structure's unit cell with parameters as follows: $H=0.36 \mathrm{~mm}, L_{1}=15 \mathrm{~mm}$, $L_{2}=4.9 \mathrm{~mm}, L_{3}=2 \mathrm{~mm}, W=0.2 \mathrm{~mm}, G_{1}=G_{2}=0.5 \mathrm{~mm}$, and $G_{3}=0.3 \mathrm{~mm}$. Figure 4 shows the embedded meander 


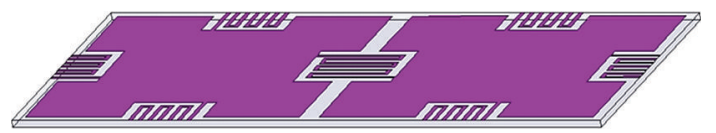

(a)

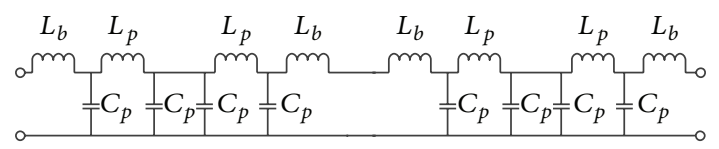

(b)

FIGURE 1: EBG structure and its equivalent circuit. (a) Two unit cells of the proposed EBG structure; (b) equivalent circuit model for the two connecting unit cells.

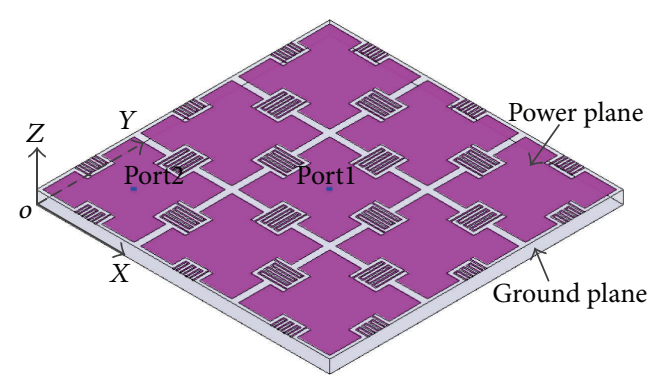

(a)

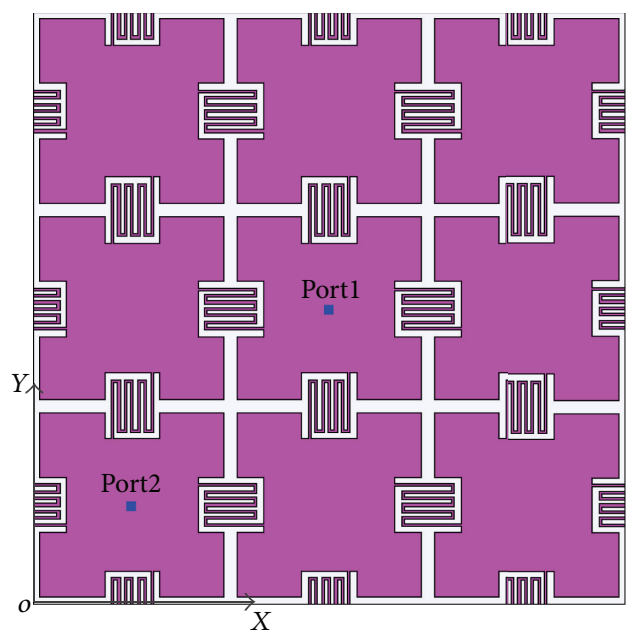

(b)

FIGURE 2: Schematic diagram of the proposed EBG structure. (a) 3D view. (b) Top view.

microstripline called bridge that connects two unit cells with parameters as follows: $L_{4}=4.5 \mathrm{~mm}, L_{5}=5 \mathrm{~mm}$.

2.3. Ultrawideband SSN Suppression. In order to evaluate the noise isolation performance of the proposed planar EBG structure, two ports, as are shown in Figure 2(b), have been set at the location of $(22.5 \mathrm{~mm}, 22.5 \mathrm{~mm})$ and $(7.5 \mathrm{~mm}$, $7.5 \mathrm{~mm}$ ) and named Portl and Port2, respectively. The left bottom corner of the board is defined as the original point of the coordinate. Simulation of the structure is carried out over

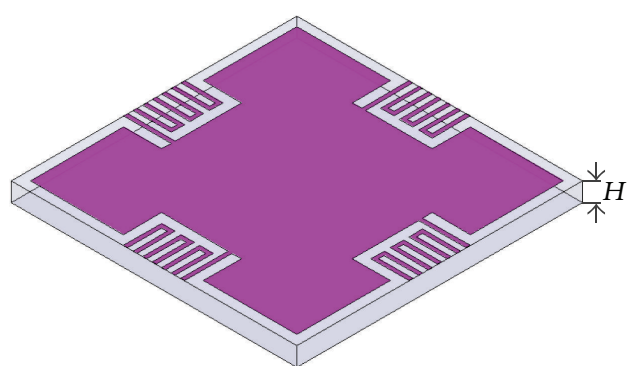

(a)

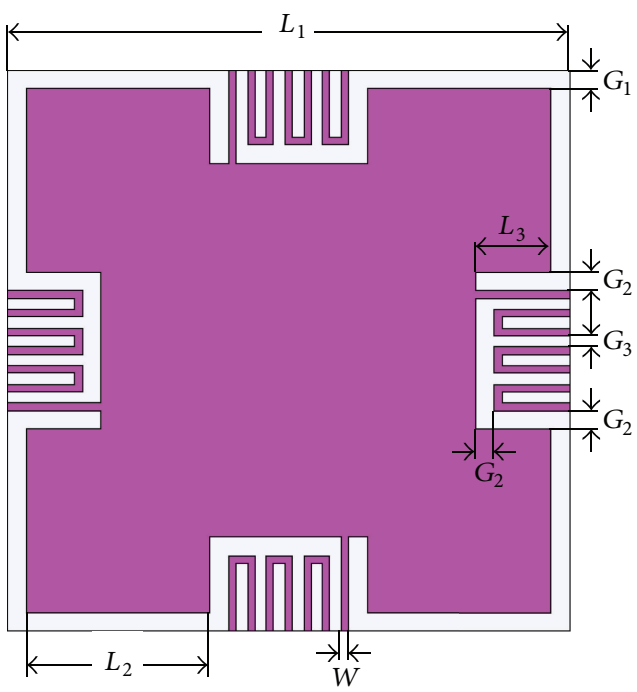

(b)

FIGURE 3: Schematic diagram of the unit cell of the proposed EBG structure and its corresponding parameters. (a) 3D view. (b) Top view.

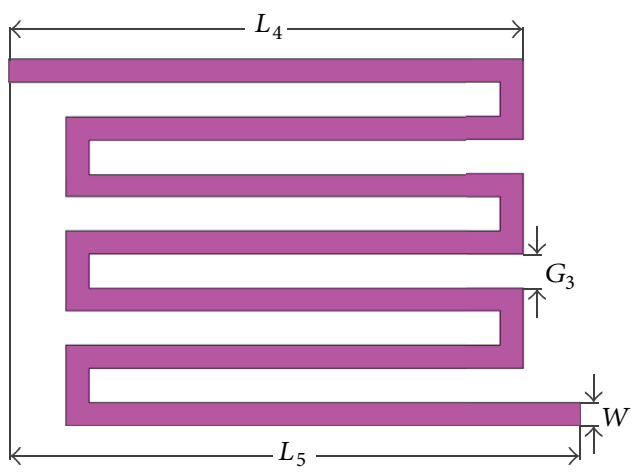

FIgURE 4: Schematic diagram of the embedded meander microstripline as bridge and its corresponding parameters.

a wide range of frequency from $40 \mathrm{MHz}$ to $40 \mathrm{GHz}$ by ansoftHFSS. The results of noise transmission coefficient $\left(S_{21}\right)$ are plotted as the blue solid line in Figure 5. For comparison, the behaviors of a reference board model with solid power and ground planes, which has exactly the same size and ports as the aforementioned EBG-patterned board, are also included as the red dashed line in Figure 5. According to the results, the stopband of the proposed EBG structure is from 


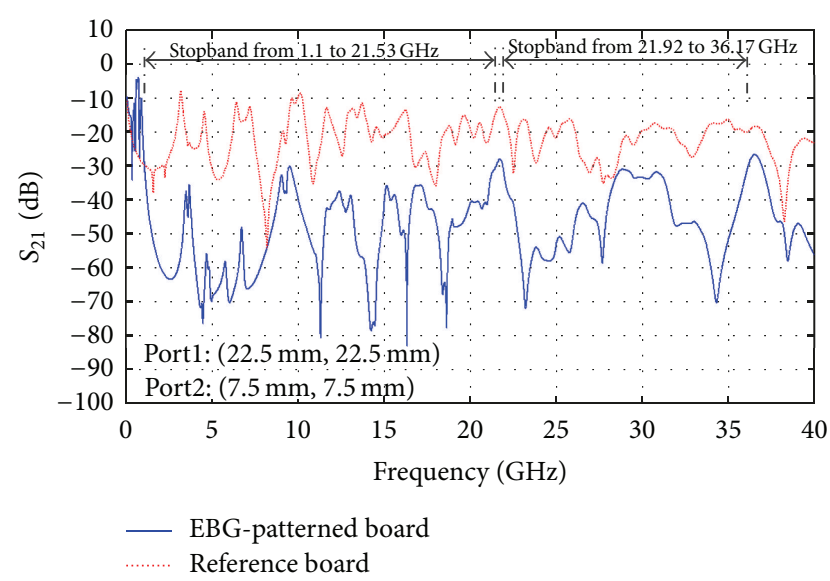

FIGURE 5: Comparison of $\left|S_{21}\right|$ between EBG-patterned board and reference board obtained by ansoft-HFSS.

1.10 to $21.53 \mathrm{GHz}$ and from 21.92 to $36.17 \mathrm{GHz}$, which covers almost the whole noise band in ultrawideband applications. Here, the stopband is defined as the frequency range of $\left|S_{21}\right|$ below $-30 \mathrm{~dB}$.

\section{Measurement}

Both the EBG-patterned board and the reference board mentioned above have been fabricated following the dimensions and requirements given before, which are shown in Figures 6(a) and 6(b), respectively. Besides, ports are added to the boards by implementing SMA as the connectors. Here, Portl $(15 \mathrm{~mm}, 15 \mathrm{~mm})$ is defined as the excitation port and Port2 $(7.5 \mathrm{~mm}, 7.5 \mathrm{~mm})$ is defined as the receiver port. The $S_{21}$ measurements of both structures have been performed by applying a Vector Network Analyzer (Anritsu 37369 C, $40 \mathrm{MHz}$ to $40 \mathrm{GHz}$ ). The measured frequency range is limited to $26.5 \mathrm{GHz}$ within which SMA can work properly. The measured data are illustrated in Figure 7, where the blue solid line represents $\left|S_{21}\right|$ of the EBG-patterned board and the red dashed line stands for the one of the reference board. Based on the results, the stopband of the proposed EBG structure ranges from 0.97 to $21.54 \mathrm{GHz}$, which is similar to the simulation results. To clearly verify the correctness of our novel design of miniaturized planar EBG structure, the measured results are compared to the simulation ones in Figure 8. Good agreement can be seen. Slight discrepancy at higher frequency may derive from the dispersion property of the FR- 4 structure and the conductor loss caused by skin effect.

\section{Eye Diagram Characterization}

Here, two different cases are compared shown in Figure 9. In the first case (structure A), the signal propagates along a microstrip line with the terminal impedance of $50 \mathrm{Ohm}$ with respect to a solid reference plane through a via down to the bottom layer and back to the top layer again. In the second case, the signal is referenced to an EBG-patterned plane

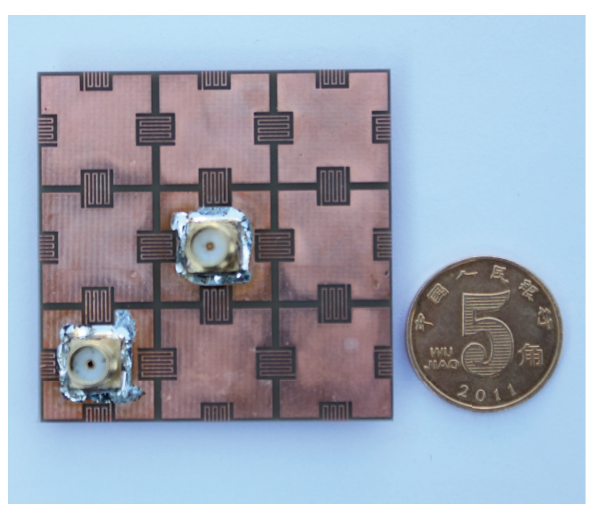

(a)

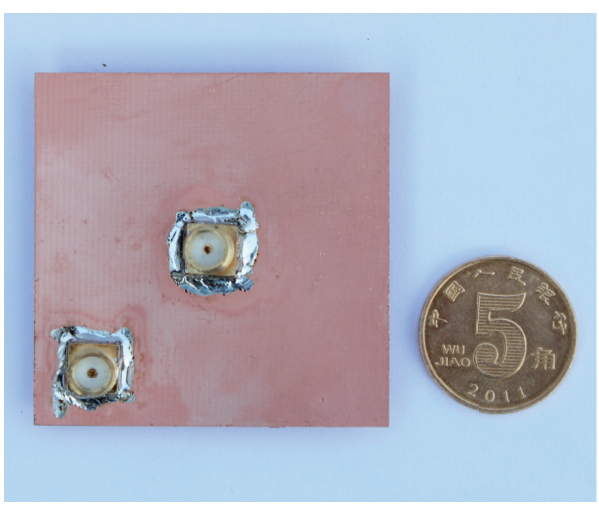

(b)

FIGURE 6: Photo of fabricated EBG test board and reference board. (a) Fabricated EBG board with two ports. (b) Fabricated reference board with two ports.

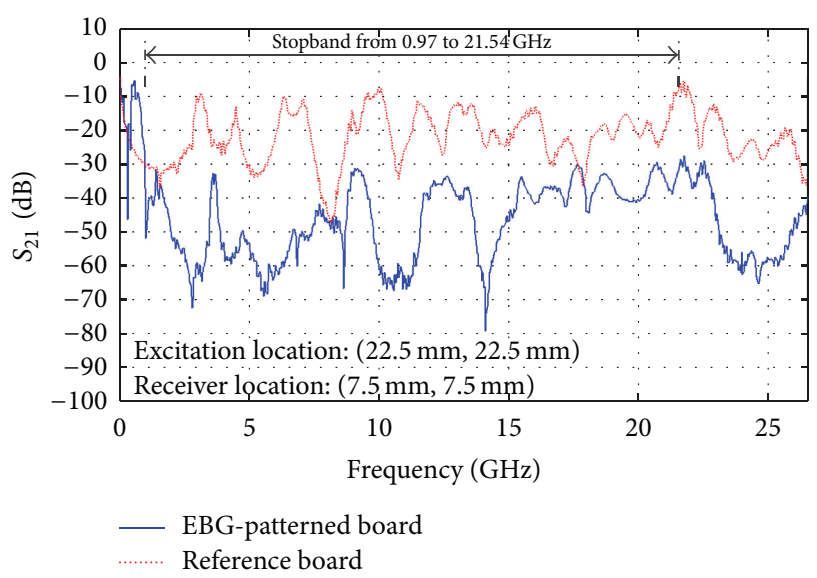

FIGURE 7: Comparison of $\left|S_{21}\right|$ between EBG-patterned board and reference board obtained by VNA.

(structure B). A nonreturn to zero (NRZ), pseudorandom binary sequence (PRBs) $2^{7}-1$ is sent at port 1 (input), and the signal propagation properties are monitored at port 2 (output). The launched PRBs are coded at $2.5 \mathrm{Gbps}$, with $500 \mathrm{mV}$ amplitude, and nominal rise/fall time is $120 \mathrm{ps}$. The eye patterns are generated by using Ansoft Designer 


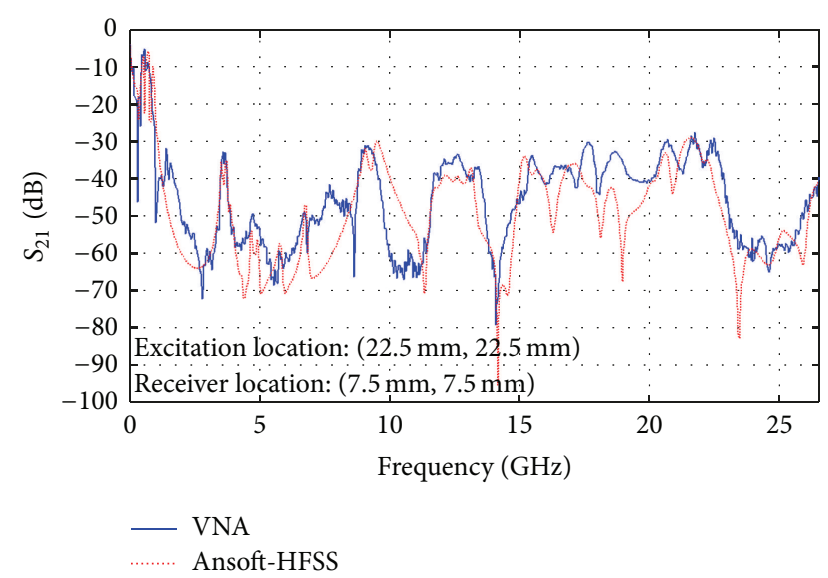

Figure 8: Comparison of $\left|S_{21}\right|$ obtained by VNA and ansoft-HFSS, respectively.

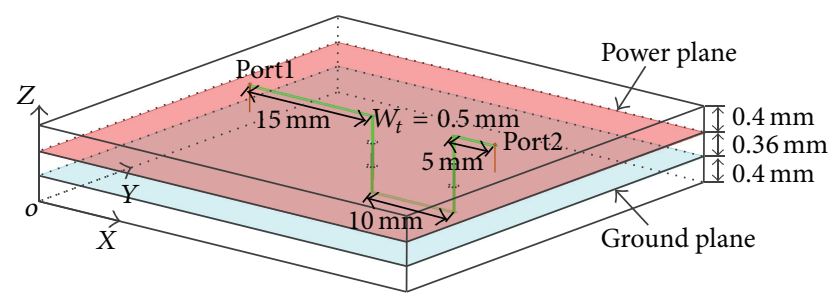

(a)

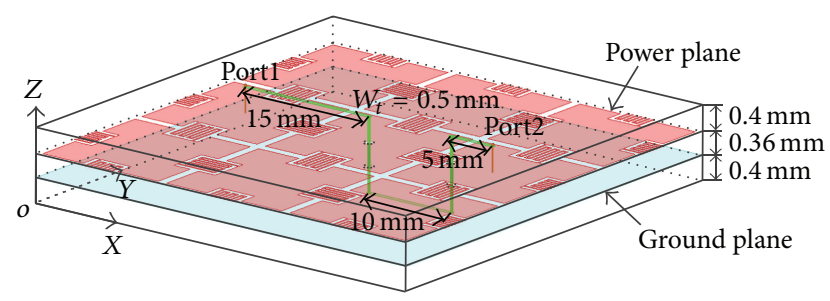

(b)

FIGURE 9: Four-layer PCBs with signal layer (a) referring to solid power plane (structure A); (b) referring to EBG patterned power plane (structure B).

convolution simulator. The simulated eye diagrams for signal streams at the output of structure A and B are shown in Figures $10(a)$ and $10(b)$. It is seen, the eye significantly shrinks for structure B. This is mainly due to the strong reflection arising from this nonuniform EBG-patterned power plane.

In order to improve the signal quality, a differential signaling strategy is adopted. In the example considered here, the traces are designed with a differential impedance of $100 \mathrm{Ohm}$ shown in Figure 11(a). The width of the lines is $0.5 \mathrm{~mm}$, and the spacing between the two lines is $0.3 \mathrm{~mm}$. The excitation signal is $2^{7}-1$ PRBs fed differentially at the two terminals of input, and eye diagram is monitored at the other ends of the differential pair as shown in Figure 11(b). It can be seen that the signal quality is greatly improved if compared with that of the single-ended feeding as shown in Figure 10(b).

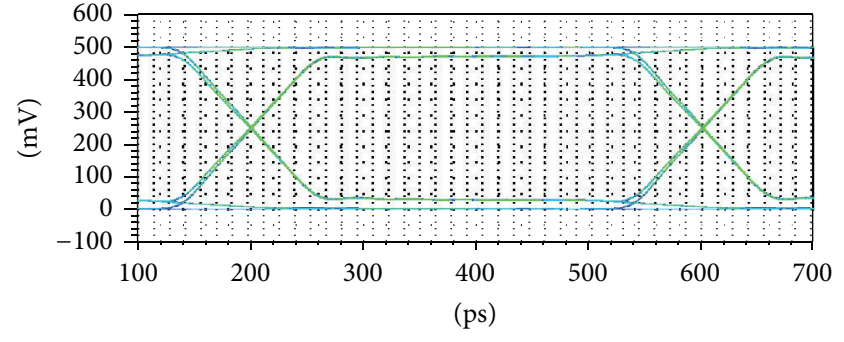

(a)

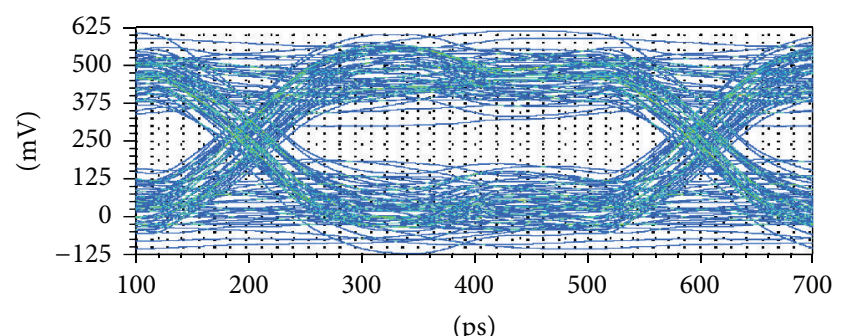

(b)

FIGURE 10: Simulated eye diagram for single-ended trace (a) solid reference board; (b) meander line EBG-patterned board.

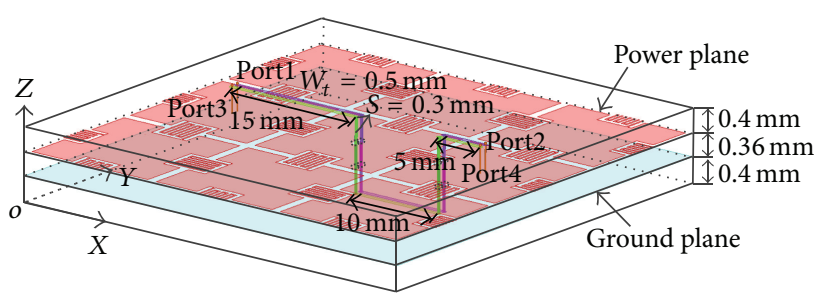

(a)

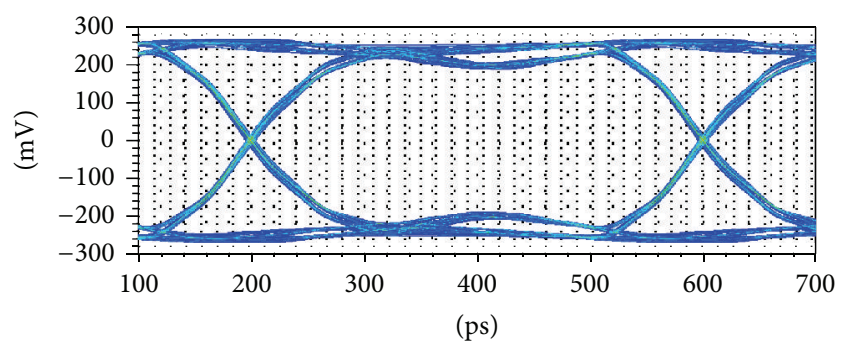

(b)

FIGURE 11: (a) Test geometry for EBG-patterned structure for differential pair configuration; (b) its corresponding eye diagram.

\section{The Suppression Effect of EBG Structure on Electromagnetic Emission}

Finally, the electromagnetic emission of the EBG board and the solid reference board is measured. There often exists electromagnetic emission on PCB boards, and theoretically speaking, the EBG structure can suppress electromagnetic emission within a certain frequency band, thus improving the radiation emission level of $\mathrm{PCB}$ and reducing the impact on the surrounding environment. The measurement results corroborate the above-mentioned theoretical prediction. 


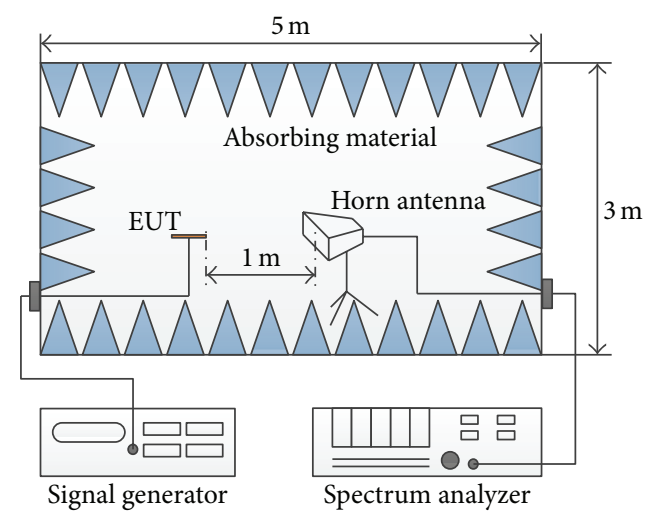

FIGURE 12: Measurement setup for EMI in $1 \mathrm{~m}$ fully anechoic chamber.

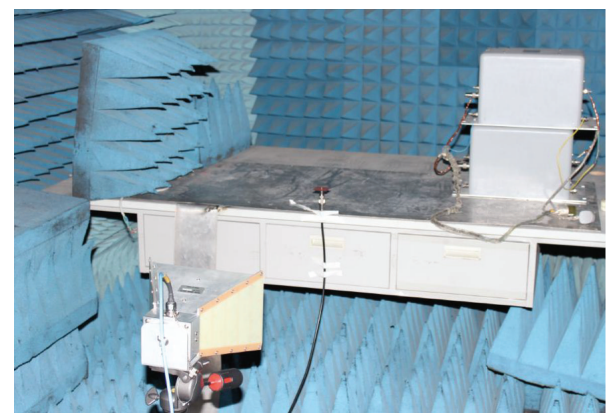

FIGURE 13: The layout photo of the test site.

This test adopts the " 1 meter" method, which means that the distance between the measurement antennas and the EUT is $1 \mathrm{~m}$. The frequency range in the measurement is $1-15 \mathrm{GHz}$ and the frequency interval of test is the $0.25 \mathrm{GHz}$. The test schematic is shown in Figure 12, and the layout of the test site is shown in Figure 13.

Figure 14 shows the measured electromagnetic emission of the EBG board and the solid reference board.

It can be seen from Figure 14 that the electromagnetic emission of the EBG structure is stronger than that of the solid reference board in $1-3 \mathrm{GHz}$. While in $3-15 \mathrm{GHz}$, the electromagnetic emission of the EBG structure, in a majority of the frequency range, is weaker than that of the solid reference board. Therefore, the EBG structure, to a certain extent, can suppress the electromagnetic emission of the PCB board within 3-15 GHz. The specific reasons are analyzed as follows.

With the signal transmitting in the EBG structure, electromagnetic wave is excited between the power and ground plane pairs due to the impedance mismatching, via switching and other reasons. For solid power/ground plane pairs, the excited electromagnetic wave can transmit to the edge of the circuit board smoothly and has a certain amount of the emitted electromagnetic wave. But for the board with EBG structure, most of the electromagnetic wave will be bound within the unit cells where the excitation source is located; thus, the electromagnetic wave emitting from the edge is reduced. So, it can be seen that in certain frequency range,

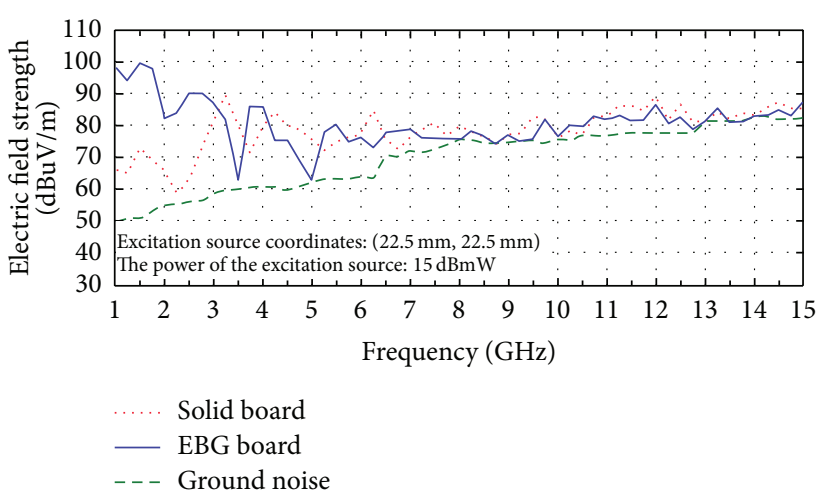

FIGURE 14: The measured electromagnetic emission of the EBG board and the solid reference board.

the electromagnetic emission of the EBG board is weaker than that of the solid reference board.

But at the same time, for the surface of the EBG structure that is provided with slots, there will be a certain amount of electromagnetic wave leaking out from the gap when the electromagnetic wavelength can be comparable to the gap length, which can be seen as a patch antenna, bringing new electromagnetic emission. So, the electromagnetic emission of the board with EBG structure is stronger than that of the solid reference board from $1 \mathrm{GHz}$ to $3 \mathrm{GHz}$.

\section{Conclusion}

Based on resonant $L-C$ circuit model, a novel miniaturized planar EBG structure with embedded meander bridge is proposed in this paper. The dimensions of the unit cell of the proposed EBG structure are $15 \mathrm{~mm} \times 15 \mathrm{~mm} \times 0.36 \mathrm{~mm}$. Measured results of noise transmission coefficient $\left(S_{21}\right)$ show good agreement with simulation ones, thus verifying the design's correctness. According to the measured results, the stopband within which $S_{21}$ is below $-30 \mathrm{~dB}$ ranges from 0.97 to $21.54 \mathrm{GHz}$. In addition, their use should be followed by careful analysis of the signal integrity on traces using the power planes as reference. The test proved that the EBG structure can suppress electromagnetic emission within a certain frequency band. The miniaturized planar EBG structure with such a wide stopband bandwidth will be very practical in ultrawideband applications.

\section{Acknowledgment}

This work was supported by the National Natural Science Foundation of China (NSFC) under Grant 61271044.

\section{References}

[1] International Technology Roadmap for Semiconductors, London, UK, 2008, http://www.itrs.net/.

[2] T. L. Wu, H. H. Chuang, and T. K. Wang, "Overview of power integrity solutions on package and PCB: decoupling and EBG isolation," IEEE Transactions on Electromagnetic Compatibility, vol. 52, no. 2, pp. 346-356, 2010. 
[3] R. Abhari and G. V. Eleftheriades, "Metallo-dielectric electromagnetic bandgap structures for suppression and isolation of the parallel-plate noise in high-speed circuits," IEEE Transactions on Microwave Theory and Techniques, vol. 51, no. 6, pp. 1629-1639, 2003.

[4] T. L. Wu, Y. H. Lin, T. K. Wang, C. C. Wang, and S. T. Chen, "Electromagnetic bandgap power/ground planes for wideband suppression of ground bounce noise and radiated emission in high-speed circuits," IEEE Transactions on Microwave Theory and Techniques, vol. 53, no. 9, pp. 2935-2942, 2005.

[5] T. L. Wu, C. C. Wang, Y. H. Lin, T. K. Wang, and G. Chang, "A novel power plane with super-wideband elimination of ground bounce noise on high speed circuits," IEEE Microwave and Wireless Components Letters, vol. 15, no. 3, pp. 174-176, 2005.

[6] Y. He, C. H. Liang, and Q. H. Liu, "Novel array EBG structures for ultrawideband simultaneous switching noise suppression," IEEE Antennas and Wireless Propagation Letters, vol. 10, pp. 588-591, 2011.

[7] J. Qin and O. M. Ramahi, "Ultra-wideband mitigation of simultaneous switching noise using novel planar electromagnetic bandgap structures," IEEE Microwave and Wireless Components Letters, vol. 16, no. 9, pp. 487-489, 2006.

[8] P. H. Rao and M. Swaminathan, "A novel compact electromagnetic bandgap structure in power plane for wideband noise suppression and low radiation," IEEE Transactions on Electromagnetic Compatibility, vol. 53, no. 4, pp. 996-1004, 2011.

[9] P. H. Rao, "Hybrid electromagnetic bandgap power plane for ultra-wideband noise suppression," Electronics Letters, vol. 45, no. 19, pp. 981-982, 2009.

[10] Y. Han, Z. Yan, Y. Wang, and T. Rahman, "A novel EBG structure with embedded meander bridge for broadband suppression of SSN," in Proceedings of the Asia-Pacific Symposium on Electromagnetic Compatibility (APEMC '12), pp. 325-328, Singapore, May 2012. 

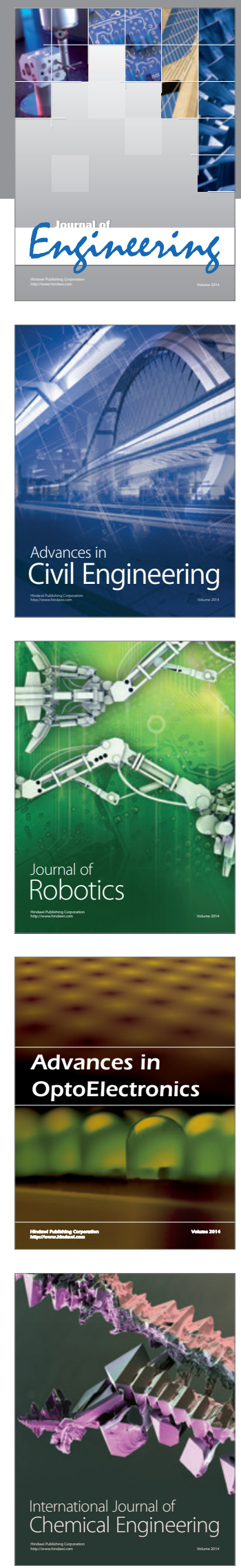

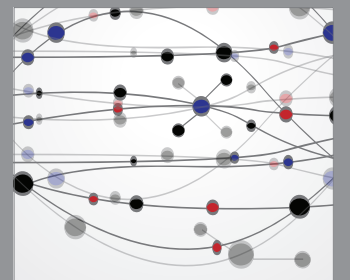

The Scientific World Journal
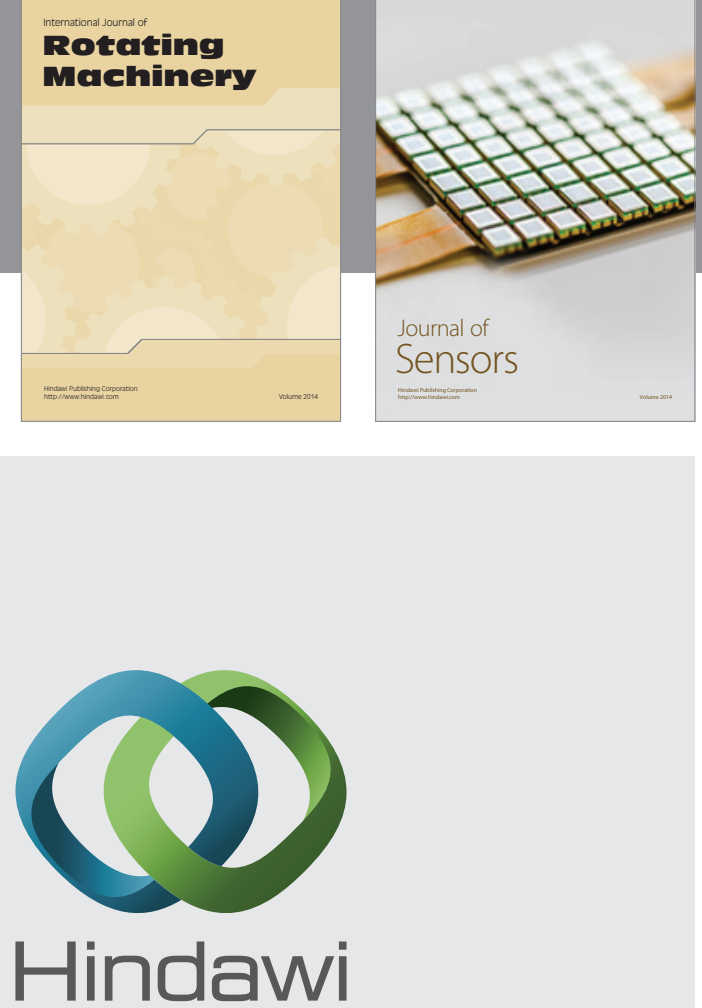

Submit your manuscripts at http://www.hindawi.com
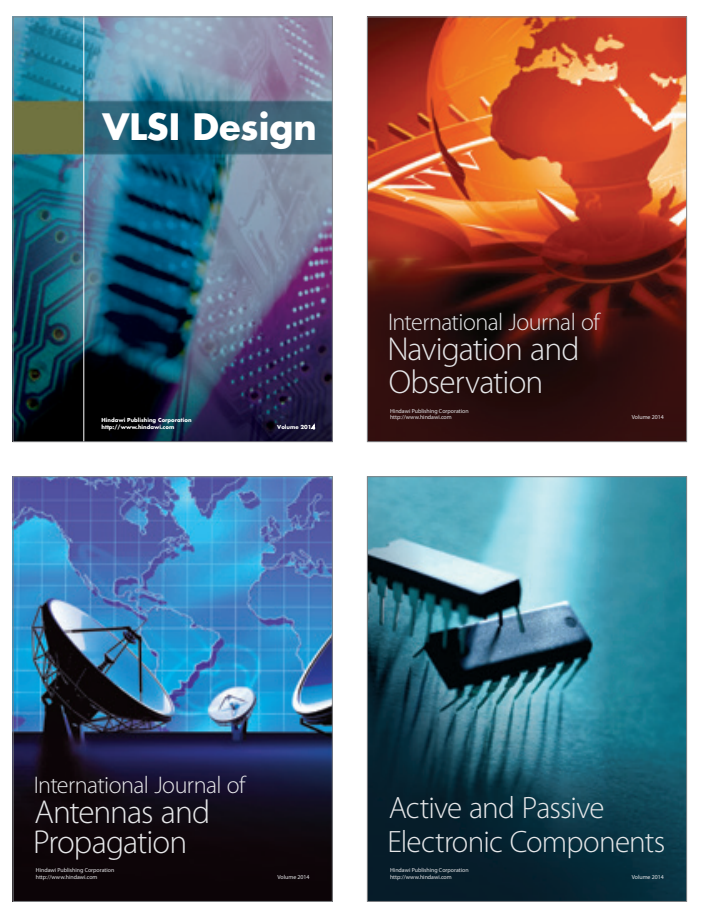
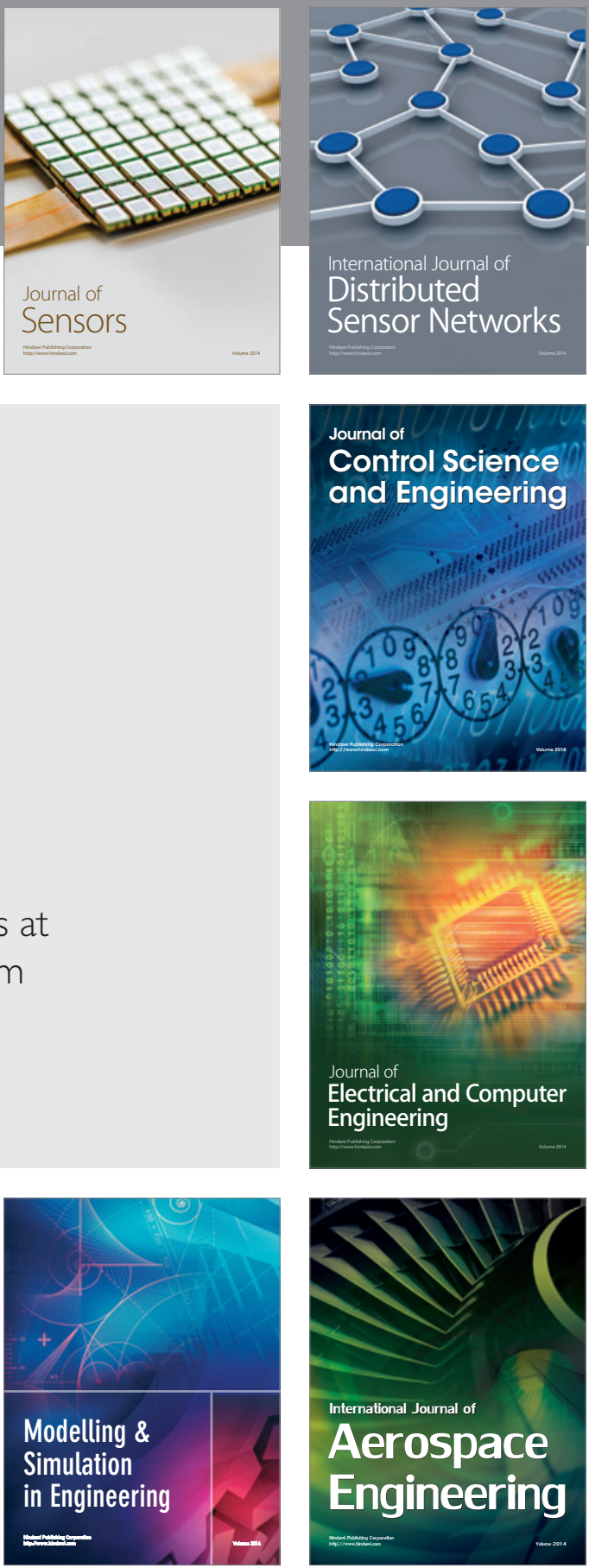

Journal of

Control Science

and Engineering
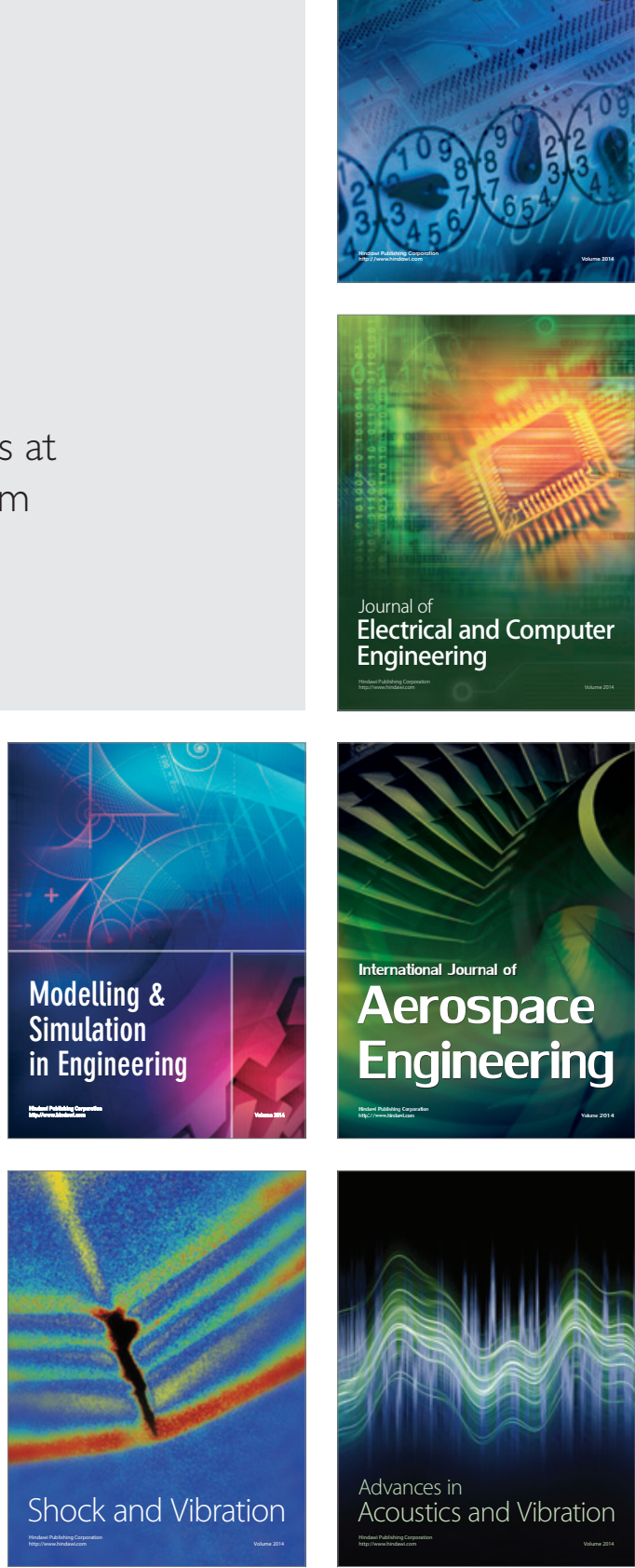